\title{
Pn Junction Temperature-Sensor by Use of Superimposed AC Signal
}

\author{
Noriaki Takashima Student Member (Tohoku Gakuin University, ) \\ Mitsuteru Kimura Member (Tohoku Gakuin University, kimura@tjcc.tohoku-gakuin.ac.jp)
}

Keywords : temperature-sensor, diode sensor, pn diode, forward voltage, $\mathrm{Si}$, ac signal

Thermistor and thermodiode are very familiar as an absolute temperature-sensor. However the thermistor has narrow measurable temperature range as a single temperature-sensor and has a non-linear relationship between the temperature $T$ and the output voltage. As to the thermodiode they have a linear dependence between forward voltage $V$ and absolute temperature $T$ under fixed forward current $I_{0}$. However, it needs the off-set balance control of the OP-amp in order to amplify the dc signal.

On the other hand our temperature-sensor uses only one pn junction diode and it is unnecessary to control the off-set voltage. It uses superimposed ac signal to the dc forward constant current and pickup the ac output signal voltage via the dc-cut capacitor connected to the pn junction diode as a temperature-sensor. It can measure the wide temperature range between $77 \mathrm{~K}$ and $400 \mathrm{~K}$ and has theoretically the output voltage proportional to the absolute temperature $T$. In addition it has merits that the calibration of our temperature-sensor should be theoretically done at only one temperature point, that temperature measurement by use of ac signal can be isolated from dc circuits for bias voltage application circuits, and that thermometry of a very small area can be achieved because of a simple and very small sensing probe of the pn junction diode.

Generally forward biased current $I$ of a pn junction diode is expressed as following equation.

$$
I=I_{s}(T) \operatorname{Exp}\left(\frac{q V}{n k T}\right)=I_{\infty} \operatorname{Exp}\left(\frac{q\left(V-V_{d}\right)}{n k T}\right)
$$

Differentiating this equation (1) by $V$, changing it into an expression to the absolute temperature $T$, putting dc current $I$ with constant value $I_{0}$ and rearranging $d I / d V$ in $\Delta I / \Delta V$ for output signal or $\delta I / \delta V$ for superimposed signal, therefore we can get following two equations,

$$
\begin{aligned}
& T=\frac{q}{n k} \frac{I_{0}}{\delta I} \Delta V=\alpha \Delta V \\
& T=\frac{q}{n k} I_{0} \delta V \frac{1}{\Delta I}=\beta \frac{1}{\Delta I}
\end{aligned}
$$

When small ac current $\delta I$ with the constant amplitude is superimposed to the constant dc diode current $I=I_{0}$, and we measure the corresponding output signal $\Delta V$ across the diode at that time, the expression equation (2) means that absolute temperature $T$ is in proportion to the amplitude of this $\Delta V$. In addition, for superimposed $\delta V$ with the constant amplitude and for measurement of the ac output signal current $\Delta I$ through the diode likewise, the expression of equation (3) means that $T$ will be inversely proportional to this $\Delta I$. Therefore, we should determine fixed value $\alpha$ or $\beta$ for equation (2) or equation (3), respectively, at single temperature point. We can achieve the extremely simple thermometer which can directly display the absolute temperature
$T$.

We have used a commercial Si pn junction diode and a $5.6 \mathrm{~V} \mathrm{Si}$ Zener diode to verify the theory, because it is unnecessary to use special diodes. In our experiments, we put the dc current $I_{0}=100$ $\mu \mathrm{A}, \delta I=10 \mu \mathrm{A}$ and $f=1 \mathrm{kHz}$. Temperature - Output Voltage characteristics of the Si diode and the Zener diode shown in Fig. 1 and Fig. 2, respectively. From Fig. 1 for the diode with superimposed small ac current $\delta I$ we can see that the relationship between the output voltage $V_{V}$ proportional to the $\Delta V$ and the absolute temperature $T$ has almost linear dependence in the temperature range between $77 \mathrm{~K}$ and $400 \mathrm{~K}$ as the theory written in equation (2) except a characteristic change appearing near the room temperature. In Fig. 2 the relationship between the output voltage $V_{V}$ proportional to $\Delta V$ and the absolute temperature $T$ for the commercial Si diode of the Zener diode as shown.

In above $400 \mathrm{~K}$ we see the sudden decrease in output voltage $V$ in the characteristics. This decrease will come from intrinsic carrier generation beyond the band gap energy. The difference in these characteristics of Fig. 1 and Fig. 2 will come from diode structures concerning especially with impurity concentrations. More linear temperature $T$ dependence of output voltage $V_{V}$ in the wide temperature range will be expected by impurity-control of the diode fabrication and by appropriate calibration of measured results.

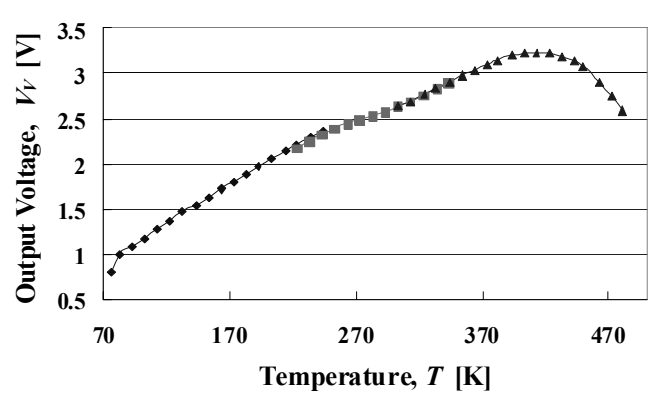

Fig. 1. Temperature $T$ dependence of output voltage $V_{V}($ Normal Si Diode $)$

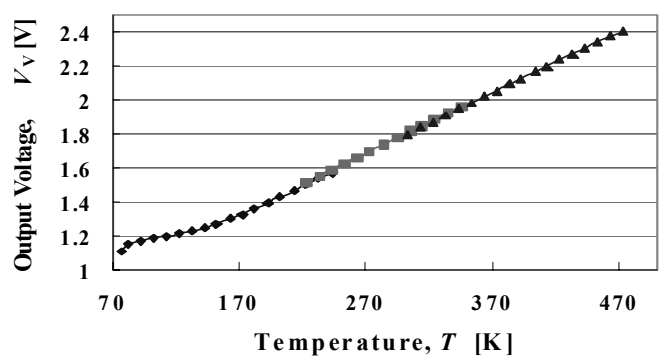

Fig. 2. Temperature $T$ dependence of output voltage $V_{V}(5.6 \mathrm{~V}$ Si Zener Diode) 


\title{
Pn Junction Temperature-Sensor by Use of Superimposed AC Signal
}

\author{
Noriaki Takashima* Student Member \\ Mitsuteru Kimura* Member
}

\begin{abstract}
A new type absolute temperature-sensor with the measurable temperature range between $77 \mathrm{~K}$ and $400 \mathrm{~K}$ based on the forward voltage change due to the ambient temperature is proposed and demonstrated. In this temperature-sensor the forward ac voltage change or the forward ac current change at the pn junction diode due to the temperature change is measured for a constant amplitude of superimposed ac small signal of current $\delta I$ or voltage $\delta V$, respectively, on the constant forward dc current $I_{0}$. This sensor has merits that it can be calibrated at only one temperature point, that output voltage is proportional to the absolute temperature $T$ and that the measurement can be done independent of the ground point, since the ac signal circuits can be isolated from de bias voltage circuits.
\end{abstract}

Keywords : temperature-sensor, pn diode, forward voltage, $\mathrm{Si}$

\section{Introduction}

Thermistor, thermodiode and IC temperature-sensor are very familiar as the absolute temperature-sensors. However the thermistor has narrow measurable temperature range as a single temperature-sensor and has a non-linear relationship between the temperature $T$ and the output voltage. As to the thermodiode and the IC temperature-sensor they have a merit to have a linear relationship between the forward voltage $V$ and the absolute temperature $T$ under a fixed forward current $I_{0}$, however, it needs the off-set balance control of the OP-amp in order to amplify the dc signal ${ }^{(1)}$. The principle of the traditional thermodiode is as follows $^{(2)(3)}$ :

$$
I=I_{s}(T) \operatorname{Exp}\left(\frac{q V}{n k T}\right)=I_{\infty} \operatorname{Exp}\left(\frac{q\left(V-V_{d}\right)}{n k T}\right),
$$

where $n, k, q, I_{s}, I_{\infty}$ and $V_{d}$ are ideality factor, Boltzmann constant, electron charge, reverse biased saturation current, diode current at $T=\infty$ and equivalent diffusion potential, respectively.

From this equation (1) we can get the following equation,

$$
V=\frac{n k T}{q} \ln \frac{I}{I_{s}(T)}=V_{d}-\frac{n k T}{q} \ln \frac{I_{\infty}}{I} .
$$

The diffusion potential $V_{d}$ has a weak temperature dependence, and will be constant as almost being the band gap potential $V_{g}$ for large impurity concentrations of both donor and acceptor. So we can neglect the temperature dependence of $V_{d}$.

In order to eliminate the temperature dependent $I_{S}(T)$ in equation (2) the temperature dependent forward voltage $V$ has to be measured using similar two pn junction diodes with different junction areas causing different constant current $I_{1}$ and $I_{2}{ }^{(4)}$. In other case using only one pn junction diode two unknown quantities, $V_{d}$ and the coefficient of temperature $T$, in equation (2) have to be determined at a fixed dc current $I_{0}$ by measurements at least at two different temperature $T_{1}$ and $T_{2}$. In Fig.1 the relationship between the forward voltage $V$ of a pn junction diode and the absolute temperature $T$ predicted from equation (2) is

\footnotetext{
* Department of Electronics, Tohoku Gakuin University

Tagajo, Miyagi 985-8537
}

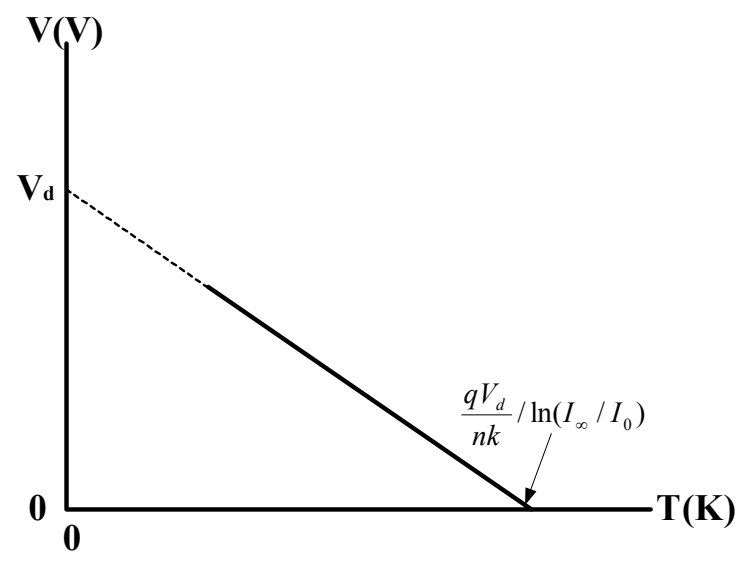

Fig. 1. Relationship between forward voltage $V$ and temperature $T$ in a pn junction diode. (Traditional thermodiode)

shown.

On the other hand, our temperature-sensor uses only one pn junction diode, and it is unnecessary to control the off-set voltage of the OP-amp because it uses superimposed ac signal to the dc forward constant current and pickups the ac output signal voltage via the dc-cut capacitor connected to the pn junction diode as a temperature-sensor. It can measure the wide temperature range between $77 \mathrm{~K}$ and $400 \mathrm{~K}$ and has the output voltage proportional to the absolute temperature $T$. In addition, it has merits that our temperature-sensor can calibrate at only one temperature point, and that thermometry of very small area can be achieved by a single pn diode with a very small junction area.

\section{Principle of the New Temperature-Sensor}

Generally forward biased current $I$ of a pn junction diode is expressed by the equation (1). Differentiating this equation (1) by $V$, changing it into an expression to the absolute temperature $T$, putting dc current $I$ with constant value $I_{0}$ and rearranging $d I / d V$ in $\Delta I / \Delta V$ for output signal or $\delta I / \delta V$ for superimposed signal, therefore we can get following two equations, 
$T=\frac{q}{n k} \frac{I_{0}}{\delta I} \Delta V=\alpha \Delta V$

where $\alpha=\frac{q}{n k} \frac{I_{0}}{\delta I}$.

$$
T=\frac{q}{n k} I_{0} \delta V \frac{1}{\Delta I}=\beta \frac{1}{\Delta I}
$$

where $\beta=\frac{q}{n k} I_{0} \delta V$.

In addition, $q$ and $k$ are the fixed numbers, and $n$ can be considered to be a constant value for once formed pn junctions.

When small ac current $\delta I$ with the constant amplitude is superimposed to the constant dc diode current $I=I_{0}$, and we measure the corresponding output signal $\Delta V$ across the diode at that time, the expression equation (3) means that absolute temperature $T$ is in proportion to the amplitude of this $\Delta V$. In addition, for superimposed $\delta V$ with the constant amplitude and for measurement of the ac output signal current $\Delta I$ through the diode likewise, the expression of equation (5) means that $T$ is inversely proportional to this $\Delta I$. Therefore, since we should decide fixed value $\alpha$ or $\beta$ for equation (3) or equation (5), respectively, at only one temperature point, we can achieve the extremely simple thermometer which can directly display the absolute temperature $T$.

\section{Experiments and Results}

We have used commercial Si pn junction diodes to verify the theory because it is unnecessary to use special diodes. In Fig.2 a block diagram of the temperature measurement circuit proportional to the output ac signal voltage $\Delta V$ under the superimposed small ac current $\delta I$ to the constant de diode current $I_{0}$ (given by equation (3)) is shown. We have used the familiar constant current circuits using an OP-amp to produce the constant dc diode current $I_{0}$ superimposed by the small ac current $\delta I$. Here, we omitted the diagram for superimposed ac signal voltage $\delta V$, because the diagram is similar except the replacement of $\Delta I$ and $\delta I$ with $\Delta V$ and $\delta V$, respectively.

In our experiments, we put the dc current $I_{0}=100 \mu \mathrm{A}, \delta V=4 \mathrm{mV}$ and $\delta I=10 \mu \mathrm{A} . \delta V$ and $\delta I$ is peak to peak voltage and current, respectively. We have used $10 \mu \mathrm{F}$ capacitor as a coupling capacitor and $f=1 \mathrm{kHz}$ as ac signal frequency so as to neglect the ac voltage drop across the capacitor and pick up only the output signal component $\Delta V$ and $\Delta I$.

In Fig. 3, the temperature $\mathrm{T}$ dependence of output voltage $V_{V}$

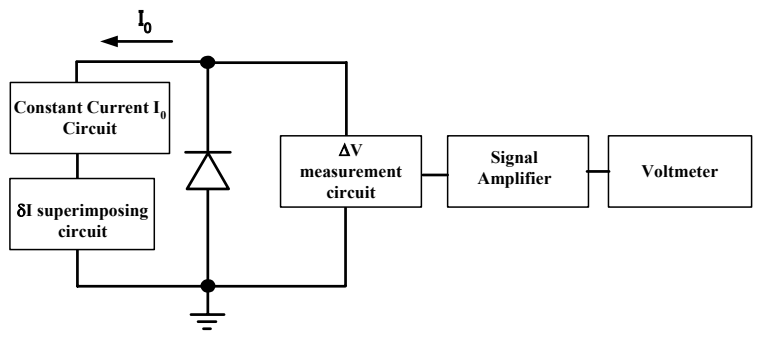

Fig. 2. Block diagram for the temperature measurement circuits proportional to $\Delta V$ corresponding to $\delta I$.

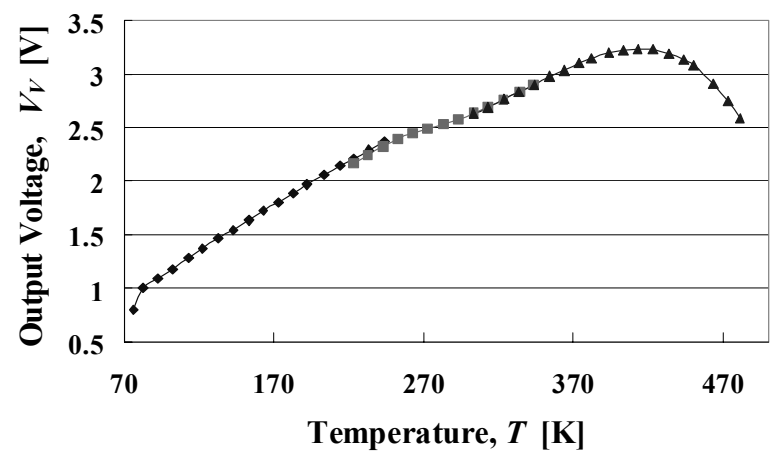

Fig. 3. Temperature $T$ dependence of output voltage $V_{V}($ Normal Si Diode).

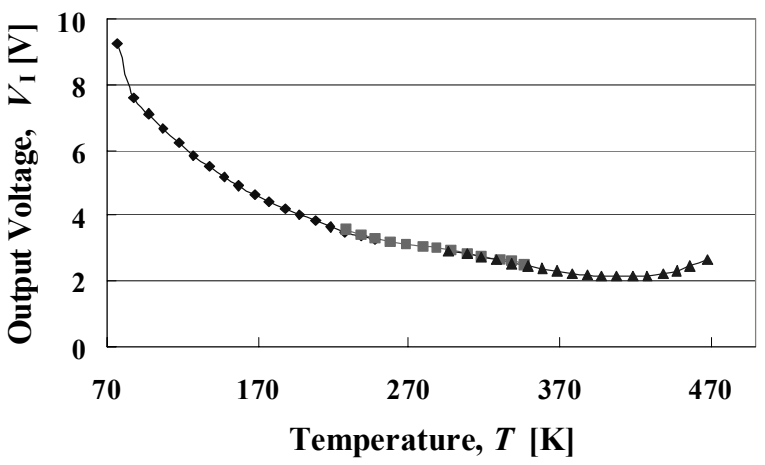

Fig. 4. Temperature $T$ dependence of output voltage $V_{I}$ (Normal Si Diode).

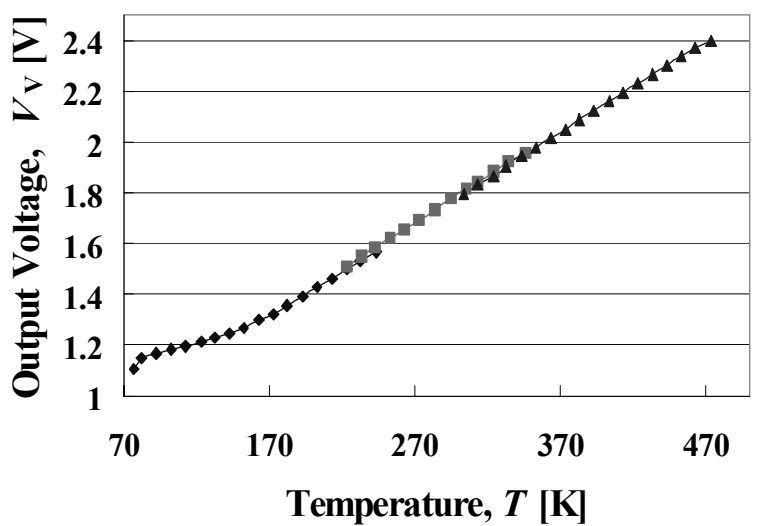

Fig. 5. Temperature $T$ dependence of output voltage $V_{V}(5.6 \mathrm{~V}$ Si Zener diode).

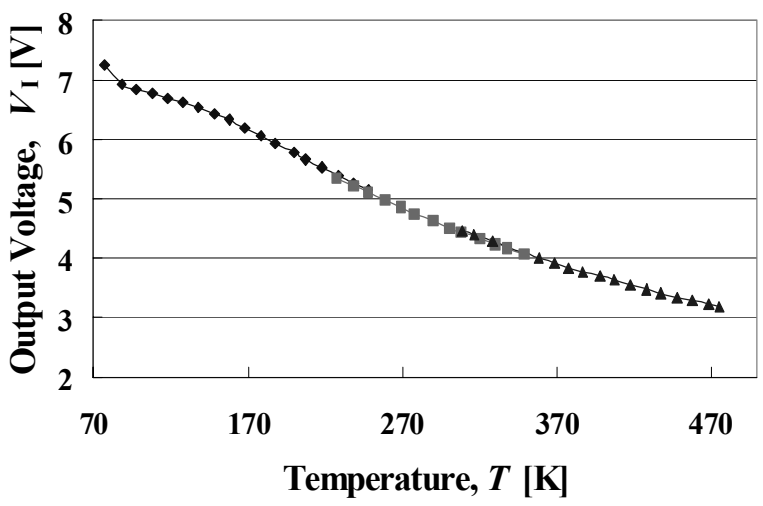

Fig. 6. Temperature $T$ dependence of output voltage $V_{I}$ (5.6V Si Zener diode). 
proportional to the $\Delta V$, which proportional to the absolute temperature $T$ (corresponding to the equation (3)), is shown.

In Fig. 4, the temperature $T$ dependence of output voltage $V_{I}$ proportional to the $\Delta I$ (corresponding to the equation (5)), is shown for same diode in Fig.3.

$V_{V}$ and $V_{I}$ represent amplified output voltages according to $\Delta V$ and $\Delta I$, respectively, by the signal amplifier shown in Fig.2.

From Fig. 3 for the diode with superimposed small ac current $\delta I$ we can see that the relationship between the output voltage $V_{V}$ proportional to the $\Delta V$ and the absolute temperature $T$ has a linear dependence in the temperature range between $77 \mathrm{~K}$ and $400 \mathrm{~K}$ as the theory written in equation (3) except a characteristic change appearing near the room temperature. As to the disturbance of characteristics appearing near room temperature it is considered that ideality factor $\mathrm{n}$ is not constant in near room temperature. In addition, we can also see that temperature $T$ dependence of the output voltage $V_{I}$ proportional to the $\Delta I$ has characteristics predicted by equation (5), in which the output voltage $V_{I}$ have relation of inverse proportion to $\Delta I$. Ideality factor $\mathrm{n}$ is estimated to be about 1.0 at $300 \mathrm{~K}$ in this normal Si diode.

In Fig.5 relationship between the output voltage $V_{V}$ proportional to $\Delta V$ and the absolute temperature $T$ for the commercial $5.6 \mathrm{~V} \mathrm{Si}$ Zener diode (corresponding to equation (3)) is shown. In Fig. 6 the relationship between the output voltage $V_{I}$ inversely proportional to $\Delta I$ and the absolute temperature $T$ for the same Zener diode (corresponding to equation (5)) is shown.

Ideality factor $n$ of this Zener diode is calculated to be about 0.7 at $300 \mathrm{~K}$ and this seems to be small. However, characteristics of the temperature $T$ dependence of the amplitude $\Delta V$ became more linear relationship as predicted in the theoretical consideration than that of the normal diode shown such as in Fig.3.

We can see from these experiments that various diodes have different values of $\mathrm{n}$ and also have different gradient changing region in $T-V_{V}$ characteristics as shown in Fig.3 and Fig.5.

\section{Discussions and Considerations}

It is well known that the value of ideality factor $n$ is 1.0 for an ideal carrier-diffusion model in a pn junction diode, and that the value will differ from 1.0 if the carrier recombination in the depletion layer and another carrier transport mechanism such as tunneling current at forward bias voltage are increased ${ }^{(4)}$. It is well known that a value of ideality factor $n$ varies between 1.0 and 2.0 for the carrier recombination mechanism in the depletion layer. A value of $\mathrm{n}$ obtained from experimental results of above Fig. 3 for the normal silicon pn junction diode is about 1.0 at $300 \mathrm{~K}$, and in this sense agreement with the theory is seen.

However, the value of ideality factor $\mathrm{n}$ is calculated to be about 0.7 at $300 \mathrm{~K}$ from results of Fig. 5 for the $5.6 \mathrm{~V}$ silicon Zener diode, and this seems very small value. This cannot be explained by a usual simple carrier recombination in the depletion layer, and it is thought that ideality factor $n$ has extremely grown big so that there is existence of different currents from diffusion current, for example, such as a tunneling current. Generally speaking the fact that the ideality factor $\mathrm{n}$ is small means that the temperature $T$ dependence of forward current $\mathrm{I}$ of the pn junction diode is small.

Since the 5.6 V Si Zener diode, which has relatively small breakdown voltage, is used in this experiment, this pn junction diode is probably composed of both heavily doped $\mathrm{p}+$ and $\mathrm{n}+$ region. It is well known that a heavily doped diode has tunneling component in forward biased current because of narrow depletion

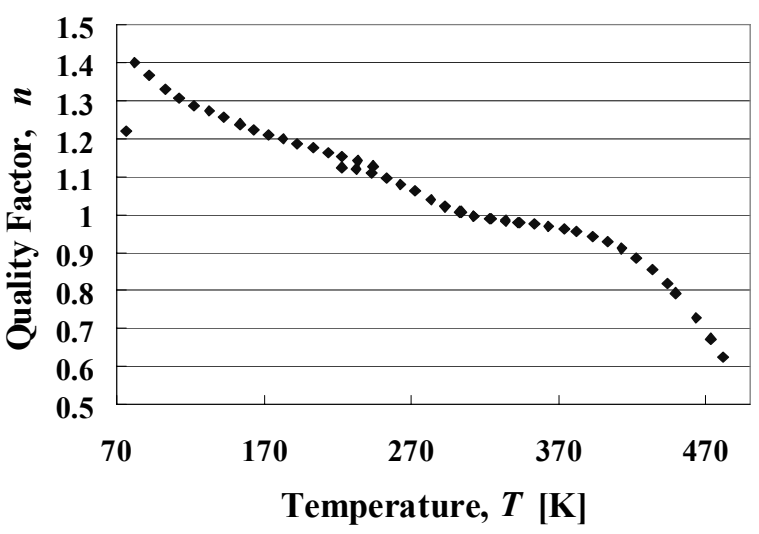

Fig. 7. Temperature $T$ dependence of the quality factor $\mathrm{n}$ calculated using the data of the normal diode shown in Fig.3.

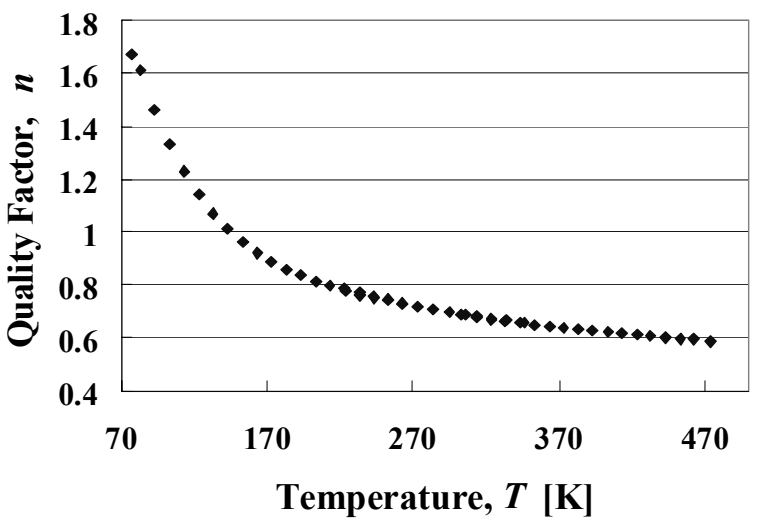

Fig. 8. Temperature $T$ dependence of the quality factor $\mathrm{n}$ calculated using the data of the Zener diode shown in Fig.5.

layer with many deep centers in the energy gap Eg. So this Zener diode may have the junction so that the tunneling current appears.

If we compare the results of the normal Si diode shown in Fig.3 and Fig. 4 with that of the Zener diode shown in Fig.5 and Fig.6, it is found that the output voltage $V_{V}$ and $V_{I}$ are smaller than that of Fig. 3 and Fig. 4 in the same temperature range even though we use the same measurement circuits. This fact may be the evidence of existence of the tunneling current component in the total forward current, and this tunnel current component will decrease the value of $n$, since the experimental total current I will be increased due to the existence of the additional currents such as the tunnel current to the diffusion current in equation (1).

In Fig. 7 and Fig. 8 the ideality factor $n$ calculated by use of the data shown in Fig.3 (the normal diode) and Fig.5 (the Zener diode), respectively, are shown as a function of measured temperature $T$.

From these Fig. 7 and Fig. 8 we can see that the ideality factor itself depend on the temperature $T$ having a declining tendency as increase of the temperature $T$. In Fig.7 a sharp decline above 400 $\mathrm{K}$ probably come from thermal carrier generation mechanism across the energy band gap Eg.

Examining the real cause of the temperature dependent ideality factor $\mathrm{n}$, we will correct the ideality factor $\mathrm{n}$ so as to be a constant value by multiplication of a temperature dependent factor obtained from measured temperature $T$. 


\section{Conclusions}

A new absolute temperature sensing system using a pn junction diode, which is based on measurement of the ac output voltage $V_{V}$ or $V_{I}$ corresponding to superimposed ac small signal $\delta I$ or $\delta V$ with constant amplitude, respectively, to the fixed forward dc current $I_{0}$ is proposed. This sensor has the measurable temperature range of about between $77 \mathrm{~K}$ and $400 \mathrm{~K}$ and has the output voltage $V_{V}$ or $V_{\mathrm{I}}$ in proportion to or in reverse proportion to the absolute temperature $T$. In this temperature range the calibration is achieved at only one point temperature. In above $400 \mathrm{~K}$ we see the decrease in output voltage $V$ in the characteristics, this decrease comes from intrinsic carrier generation beyond the band gap energy. We have found the ideality factor $\mathrm{n}$ itself has temperature dependence, which has a declining tendency as temperature increase. This temperature dependence of $n$ may be overcome by adoption of larger current value of the fixed forward current $I_{0}$. If we choose the pn diode made of the wider band gap semiconductor than silicon such as $\mathrm{SiC}$, we can measure higher temperature range than that of $\mathrm{Si}$.

\section{Acknowledgement}

This work has been funded by the project of Intelligent Cluster.

(Manuscript received July 27, 2006, revised Dec. 11, 2006)

\section{References}

(1) G. C. M. Meijer and A. W. Herwaarden (Editors) : "Thermal Sensors", Institute of Physics Publishing, pp.116-120 (1994)
(2) M. Kimura and K. Toshima : "Thermistor-like pn junction temperatureSensor with variable sensitivity and its combination with a micro-air-bridge heater", Sensors and Actuators, A108, pp.239-243 (2003)

(3) N. Ichinose and T. Kobayashi : "Sensors and it applications", sougoudenshi-syuppan, pp.119-122 (1984)

(4) S. M. Sze (Editors) : "Semiconductor Sensors", John Wiley \& Sons, Inc., pp.370-372 (1994)

(5) S. M. Sze : "Physics of Semiconductor Devices", John Wiley \& Sons, Inc., pp.102-104 (1969)

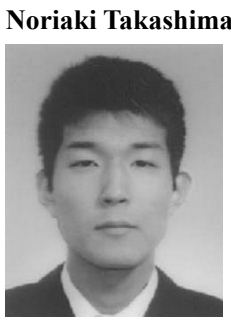

(Student Member) He was born in Yamagata, Japan in 1984. He received the B.S degree in electricity \& Information Technology. from Tohoku-Gakuin University in 2006, and currently is a graduate student of the same university. He is engaged in research on sensor devices.

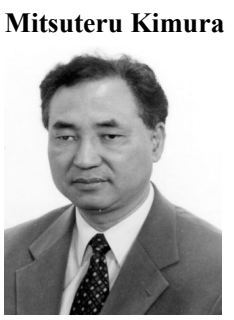

(Member) He was born in Akita, Japan in 1942. He received the B.S degree in electronics from University of Electro-Communications in 1967, and the M.S and Ph. D degrees in also electronics from Tohoku University in 1969 and 1974, respectively. He joined Tohoku-Gakuin University in 1974, and is currently a Professor in the department of electronics. He is currently engaged in research on semiconductor devices, optical devices and sensor devices. 